\title{
REVIEWS \\ The "Waze" of Inequity Reduction Frameworks for Organizations: a Scoping Review
}

\author{
Sivan Spitzer-Shohat, $\mathrm{PhD}^{1,2}$ and Marshall H. Chin, MD, MPH ${ }^{2,3}$ \\ 'Department of Population Health, Azrieli Faculty of Medicine, Bar-llan University, Safed, Israel; ${ }^{2}$ Center for Health and the Social Sciences, University \\ of Chicago, Chicago, IL, USA; ${ }^{3}$ Section of General Internal Medicine, University of Chicago, Chicago, IL, USA.
}

BACKGROUND: Different conceptual frameworks guide how an organization can change its policies and practices to make care and outcomes more equitable for patients, and how the organization itself can become more equitable. Nonetheless, healthcare organizations often struggle with implementing these frameworks.

OBJECTIVE: To assess what guidance frameworks for health equity provide for organizations implementing interventions to make care and outcomes more equitable. STUDY DESIGN: Fourteen inequity frameworks from scoping literature review 2000-2017 that provided models for improving disparities in quality of care or outcomes were assessed. We analyzed how frameworks addressed key implementation factors: (1) outer and inner organizational contexts; (2) process of translating and implementing equity interventions throughout organizations; (3) organizational and patient outcomes; and (4) sustainability of change over time.

PARTICIPANTS: We conducted member check interviews with framework authors to verify our assessments.

KEY RESULTS: Frameworks stressed assessing the organization's outer context, such as population served, for tailoring change strategies. Inner context, such as existing organizational culture or readiness for change, was often not addressed. Most frameworks did not provide guidance on translation of equity across multiple organizational departments and levels. Recommended evaluation metrics focused mainly on patient outcomes, leaving organizational measures unassessed. Sustainability was not addressed by most frameworks.

CONCLUSIONS: Existing equity intervention frameworks often lack specific guidance for implementing organizational change. Future frameworks should assess inner organizational context to guide translation of programs across different organizational departments and levels and provide specific guidelines on institutionalization and sustainability of interventions.

KEY WORDS: inequities; disparities; organizational change; frameworks; implementation.

J Gen Intern Med 34(4):604-17

DOI: $10.1007 / \mathrm{s} 11606-019-04829-7$

(c) Society of General Internal Medicine 2019

Received August 9, 2018

Revised October 29, 2018

Accepted December 28, 2018

Published online February 7, 2019

\section{BACKGROUND}

Healthcare organizations have aimed to reduce inequities in access and quality of care for over 30 years. ${ }^{1}$ While some efforts have shown success in minimizing disparities, ${ }^{2,3}$ others have documented stagnation or even exacerbation. ${ }^{4,5}$ Analogous to the Waze driving navigation software, different conceptual frameworks guide how an organization can change its policies and practices to make care and outcomes more equitable for patients, and how the organization itself can become more equitable. Nonetheless, healthcare organizations often struggle with implementing these frameworks. ${ }^{6-9}$

Change is complex due to the multifaceted nature of healthcare organizations, and because equity-focused frameworks call on healthcare organizations to simultaneously address the needs of both patients and employees. Comprehensive equity efforts require healthcare providers to tailor access and processes of care to different population groups, while also changing internal processes to promote diversity and inclusion within the organization, such as through employee hiring and retention. Interventions require changing policies, processes, and practices throughout multiple levels of the organization. ${ }^{10}$,

${ }^{11}$ Few healthcare organizations engage in comprehensive, multifaceted efforts to improve equity. ${ }^{12}$

Organizational change in general is difficult:; ${ }^{13}{ }^{14}$ literature indicates that over $70 \%$ of organizations do not achieve their aspired change goals. ${ }^{15}$ Major obstacles include the difficulty of changing the organizational culture and environment, carving out a new course when the organization seems to be functioning well, and planning and executing implementation. ${ }^{16}$ Barriers arise from institutionalization, in which the existing cognitive activities and regulative elements in the organization and its environment are resilient. ${ }^{10}$ People's intentions, actions, and rationality are conditioned by the institution they wish to change. ${ }^{17}$ Incorporating change within healthcare organizations is especially difficult because of the highly professionalized setting and embedded policy legacies, constituencies, and structured processes. ${ }^{16,}{ }^{18}$ Implementing equity frameworks requires transformational change. All organizational levels and professional backgrounds, such as administrative and clinical staff as well as patients, must participate to adapt to the shifting environment. ${ }^{19,20}$

This paper reviews and assesses what guidance frameworks for health equity provide for organizations implementing 
interventions to make care and outcomes more equitable. The objectives are twofold: (a) Identify existing frameworks that aim to assist organizations in reducing inequities in patient care and outcomes, and (b) assess to what extent the frameworks address key organizational change elements. ${ }^{21,22}$

\section{METHODS}

We conducted a scoping review of the literature. Scoping reviews map key concepts, evidence, and gaps in research by systematically searching, selecting, and synthesizing existing knowledge. ${ }^{23,} 24$ This method can address broader and more heterogeneous questions than systematic reviews. Scoping reviews can include studies of many different methodological designs, not necessarily assessing their quality. ${ }^{25-27}$

\section{Search Strategy}

We searched the following health and social science databases for the years 2000-2017: Medline, CINAHL, PsycInfo, Sociological Abstracts, and Cochrane. We also manually searched relevant websites. References from relevant articles were scanned to identify other sources Table 1.

\section{Analysis}

We assessed the frameworks utilizing Armenakis and Bedeian (1999) and Pettigrew and colleagues' (2001) analytical underpinnings for reviewing organizational change literature (Fig. 1). ${ }^{21,}{ }^{28}$ Our conceptual model addresses the four major multilevel and multifaceted constructs of organizational change processual analysis: context, process, outcomes, and time (Fig. 1). ${ }^{29}$

Context. Collective and individual agency drive change processes, yet these actions cannot be understood without understanding the pre-existing outer and inner organizational contexts that affect information, insight, and influence. Outer organizational context includes the economic, social, political, competitive, and sectoral environments in which the organization is located. It includes, for example, the economic status

\section{Table 1 Search Strategy}

\footnotetext{
A. Electronic Databases - databases were searched using the following search terms and search string:

1. framework OR roadmap OR model OR "action model" [title, abstract and keywords]

2. disparities* OR *equity [title, abstract and keywords]

3. "healthcare organizations" OR "healthcare services" OR "healthcare provider" [title, abstract and keywords] 4. 1 AND 2 AND 3

B. Websites manually searched:

American Hospital Association, Centers for Medicare and Medicaid Services, Joint Commission, National Quality Forum, Agency for Healthcare Research and Quality (AHRQ); International Health Promoting Hospitals (HPH) Network, VicHealth - Victorian Health Promotion Foundation, Close the Gap - Department of Health Australia, NHS Equality and Health Inequalities Hub
}

of surrounding neighborhoods, the demographics of the patient population, and place of residence. Additionally, the outer context also reflects powerful national policies and payment structures, such as fee for service reimbursement, valuebased payment, and national health insurance coverage. ${ }^{30}$

Inner organizational context relates to the interplay between the organizational structure and culture, including employees' readiness for change, differential needs and structures of organizational department or units, and existing knowledge and skills of employees. Organizational culture impacts employees' perceptions of the need for organizational change, innovation, diversity and equity, and their ability to carry out change. For example, a hierarchical organization culture will require topdown change directed by executive leadership, making it difficult for bottom-up change efforts by frontline employees to succeed.

The interaction between outer and inner contexts shapes the implementation process, enabling or constraining change. ${ }^{21}$

Process. Organizational change processes, conceived and initiated at the macro top management organizational level, are implemented in and across local units. ${ }^{31}$ Process analysis attempts to understand how the initiative is translated and diffused across multiple departments as well as throughout the different levels from management to frontline staff. Levels of process analysis include the organization (macro), the department or unit (meso), and individual (micro) levels. ${ }^{28}$ This analysis focuses on the way in which people understand, modify, add, or deflect organizational change throughout its diffusion across and within units of the organization, assessing the relationship between what exists and what is created. ${ }^{32}$ An example of such a translation process is organizational "sensemaking," a process of social construction in which individuals attempt to interpret and explain a set of cues, such as change initiatives, to create a plan of action for dealing with uncertainty or ambiguity. ${ }^{33,34}$

Outcomes and Time. Ultimately, organizational change is assessed through outcome performance measures. Continuous monitoring of outcomes enables understanding change not as a snapshot process, but rather as a continuous cycle over time. ${ }^{21}$

We assessed inequity reduction frameworks for their consideration of context, process, outcomes, and time. Specifically, we examined and rated on a three-point scale (do not address, partially address, and address), the extent that these frameworks considered the following: (1) Embeddedness of change in multiple contexts, focusing on the outer organizational context of the surrounding environment, and the inner organizational context of structure and culture, ${ }^{12,} 35$ (2) process of implementing change across multiple organizational settings, and guidance on translation and organizational "sense making" of the equity intervention; ${ }^{36}$ (3) impact of change processes on performance measures, including clinical and organizational outcomes, such as change in organizational culture and hiring practices; (4) sustainability of change over time. ${ }^{37}$ Authors rated the different frameworks according to 


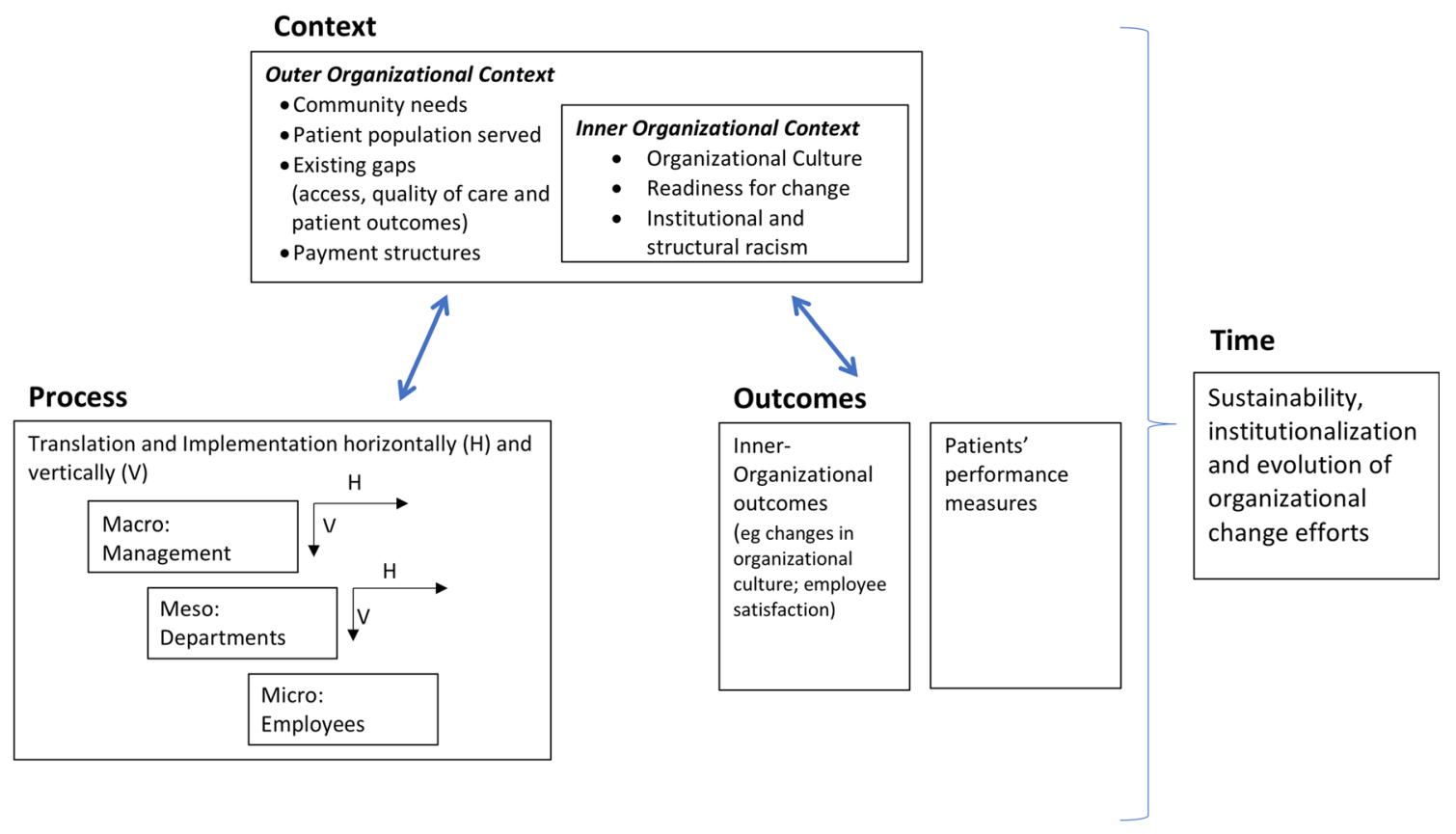

Figure 1. Conceptual organizational change model guiding assessment of reviewed frameworks addressing equity. ${ }^{21,28}$

the assessment criteria and discussed differences in ranking to reach consensus.

\section{Member Check with Authors of Frameworks/ Models}

After we identified and assessed the inequity reduction frameworks, we emailed and/or telephoned the authors and/or organizations who created them, requesting a short phone interview to conduct a member check of the findings to improve the accuracy and validity of our review. ${ }^{38}$ Prior to interview, authors were sent a draft of the manuscript and assessment table. During the interview, authors were asked to explain their framework. The assessment table was then reviewed together so that authors may have the opportunity to express their agreement or disagreement with our assessment as well as add additional insight. The study was exempt from institutional review board at both Bar-Ilan University and the University of Chicago.

\section{RESULTS}

As shown in the Preferred Reporting Items for Systematic Reviews and Meta-Analysis (PRISMA) diagram in Figure 2, a total of 2439 records were identified. Duplicate records were removed. The remaining 2065 records were screened by one of the authors (SS) for inclusion including focus on disparities or inequities and provision of a practical model, roadmap, or framework for improving access and/or quality of care. Records were excluded if the study documented or evaluated only a specific intervention and not an organization-wide initiative, or only the state of disparities. Of the records screened, 155 full texts were retrieved and assessed for eligibility.
We initially found 13 different models and frameworks addressing inequity reduction. We conducted 9 interviews verifying with authors our understanding of their frameworks according to the organizational change constructs. ${ }^{20,39-47}$ Four authors/organizations did not reply. During the member check process, one author noted an updated model that we included in this review, leading to a final set of 14 models to analyze.

Ten of the 14 models were from the United States (US). Additional frameworks were from the European Union (EU) (1), United Kingdom (UK) (1), and Australia (2). Models were developed by government (United States Department of Health and Human Services Office of Minority Health, Agency for Healthcare Research and Quality, National Health Service (NHS), Department of Health Victoria, Australia), ${ }^{39,}$ 48-50 healthcare associations (Health Promoting Hospitals Network, American Hospital Association), ${ }^{43,} 47$ not-for-profit organizations (National Quality Forum, Institute for Healthcare Improvement, Kaiela Institute), 42, 46, 51, 52 and academia. ${ }^{20,40,41,}$ ${ }^{4}$ Most frameworks addressed equity efforts through a strategy focused mainly on implementation and improvement of organizational cultural competence.

Tables 2 depicts our evaluation of frameworks according to organizational change constructs, assessing how they relate to context, process, outcomes, and time.

\section{Context}

Most frameworks primarily focused on the organization's outer context, stressing the importance of conducting a needs assessment for tailoring strategies for change. The main strategy for identifying existing disparities and population needs is analyzing demographic and performance data across patients' race and ethnicity (e.g., whether patients are predominantly African- 


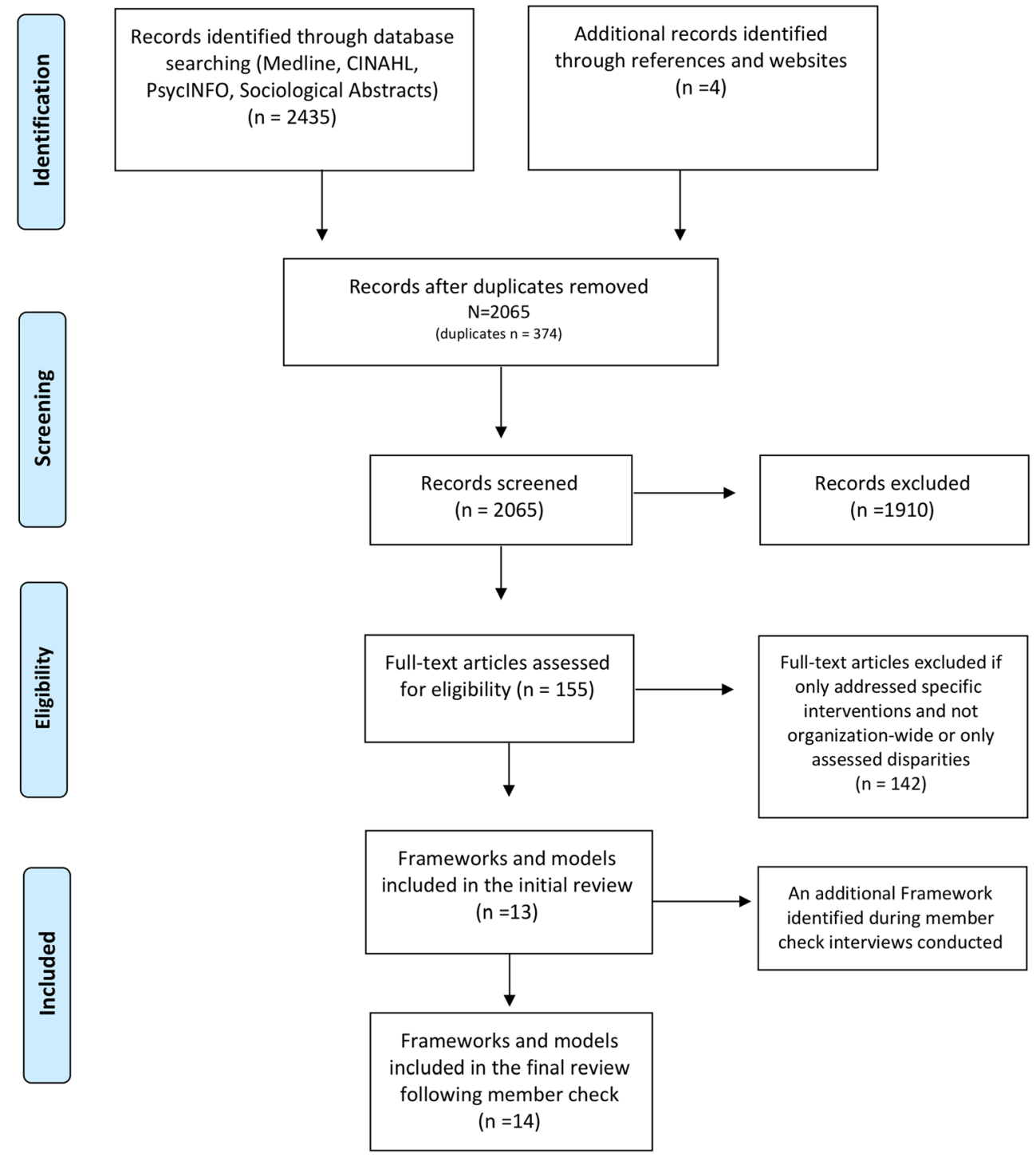

Figure 2. PRISMA flow diagram.

American or Latina, what is the percentage of non-English speakers who may require language assistance, clinical performance data stratified by patients' race and ethnicity). Additional strategies include discussions with local community representatives about their perspectives and needs, assessment of service gaps and wider social determinants of health, and appraisal of existing community resources and agencies providing care. The Equity Standards framework presents a unique approach, as it focuses not on the population but on the individual patient through the "lifeworld" approach. This framework stresses that care needs should not be assessed generally through standardized quality improvement indicators, but rather through a person-centered approach understanding individual patients' needs. ${ }^{43}$ Most frameworks do not address the effect of payment structures on healthcare organizations' outer context, how payment may affect equity, and the extent to which payment reform may be needed. The Achieving Health Equity framework encourages organizations to review payment models and suggests that a bundled payment model would account for the healthcare needs of marginalized populations. ${ }^{46}$

The inner organizational context of structure and culture was often not addressed. A few models suggested broadly that organizations assess existing resources to understand advantages and limitations, or create baseline measures of organizational performance ${ }^{20,42,50}$. The National Culturally and Linguistically Appropriate Services Standards (CLAS) Standards framework and the Disparities Leadership Program recognize the importance of existing culture. ${ }^{41,} 48$ In addition, CLAS suggests conducting an audit to assess the degree of cultural competence of employees prior to implementation. ${ }^{48}$ Few frameworks explicitly assess and discuss structural and institutional racism as part of implementation. ${ }^{46}$

\section{Process}

Most of the frameworks address the implementation process at the macro level of the whole organization. Common strategies 


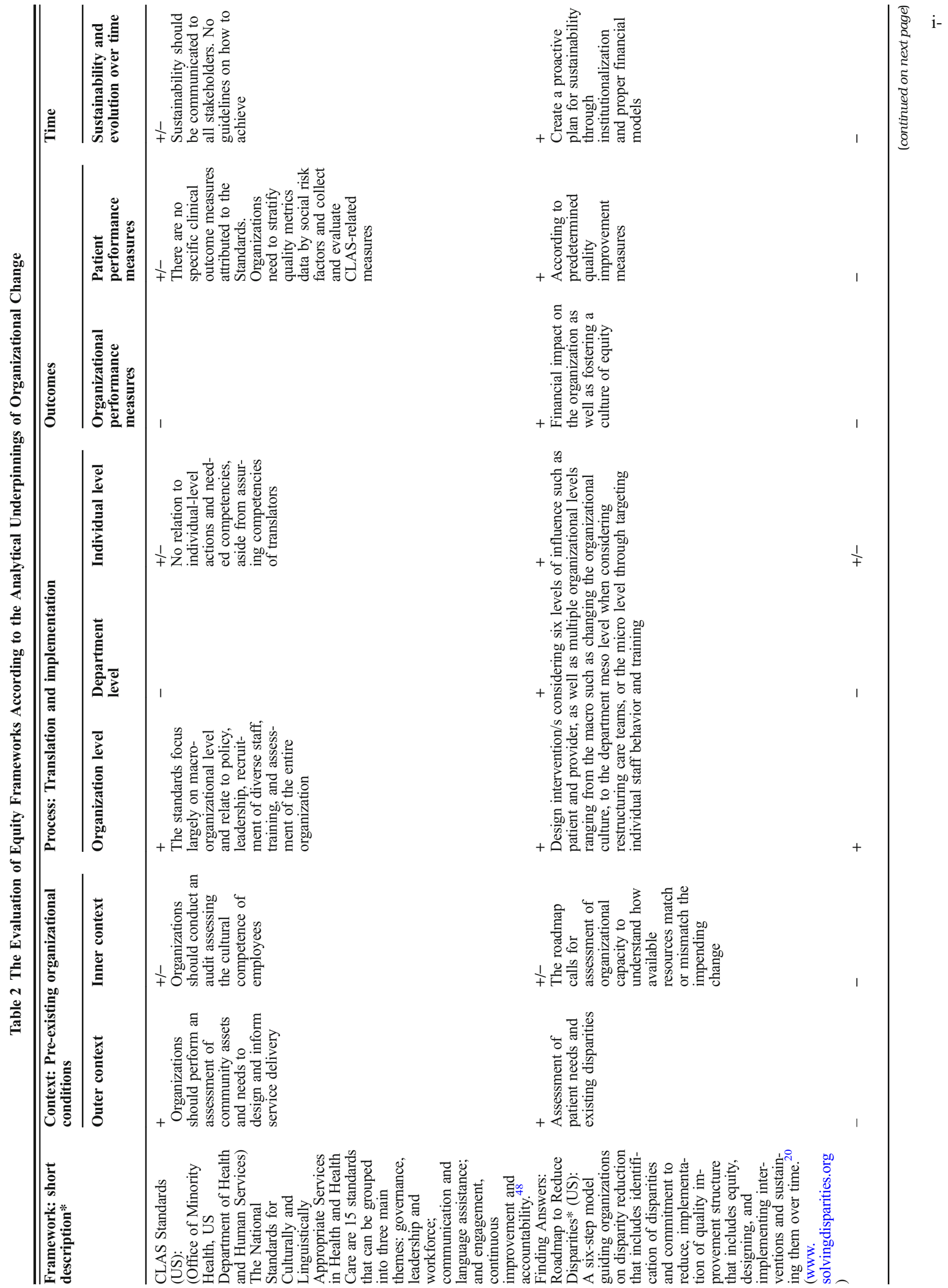




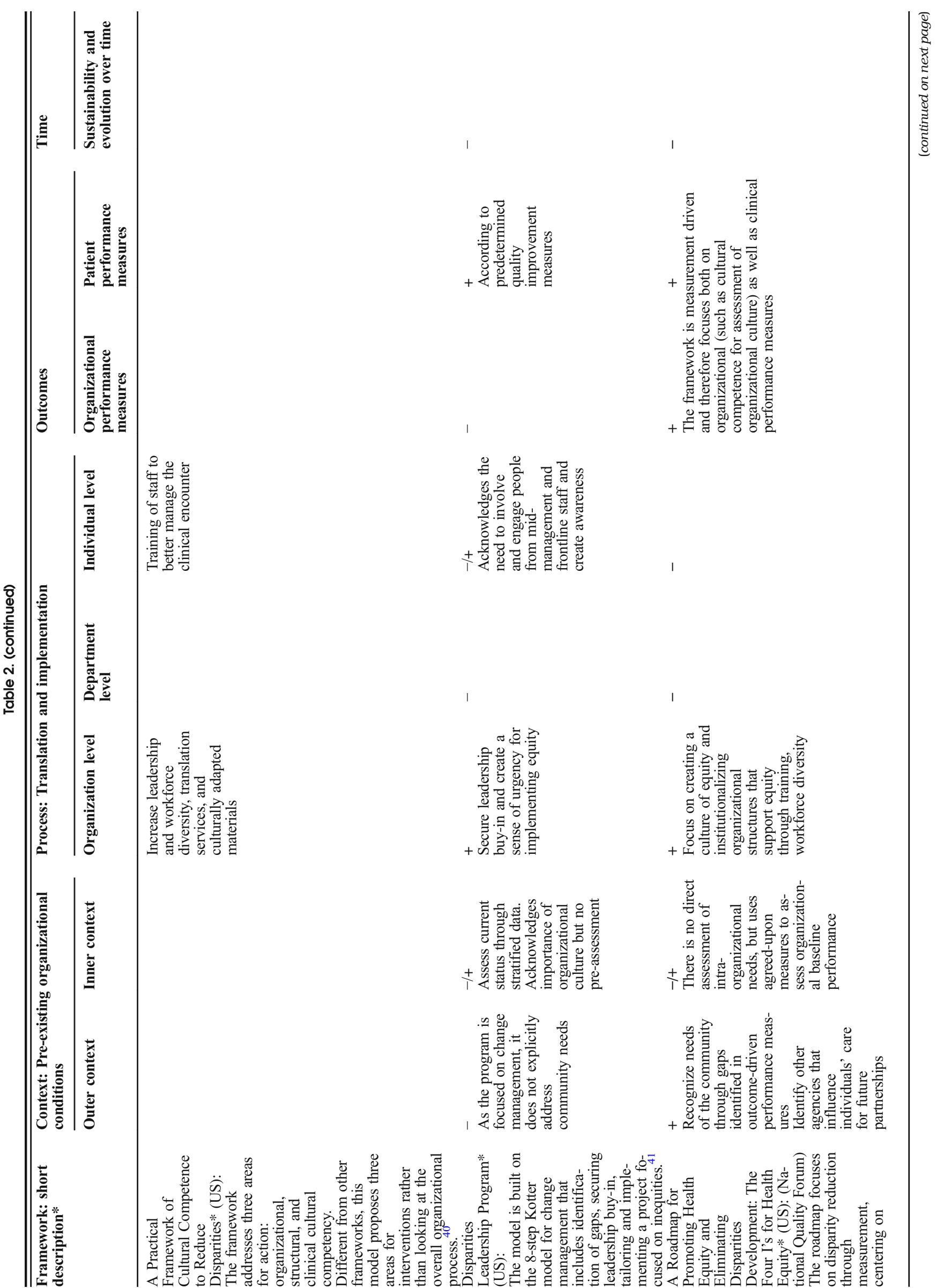



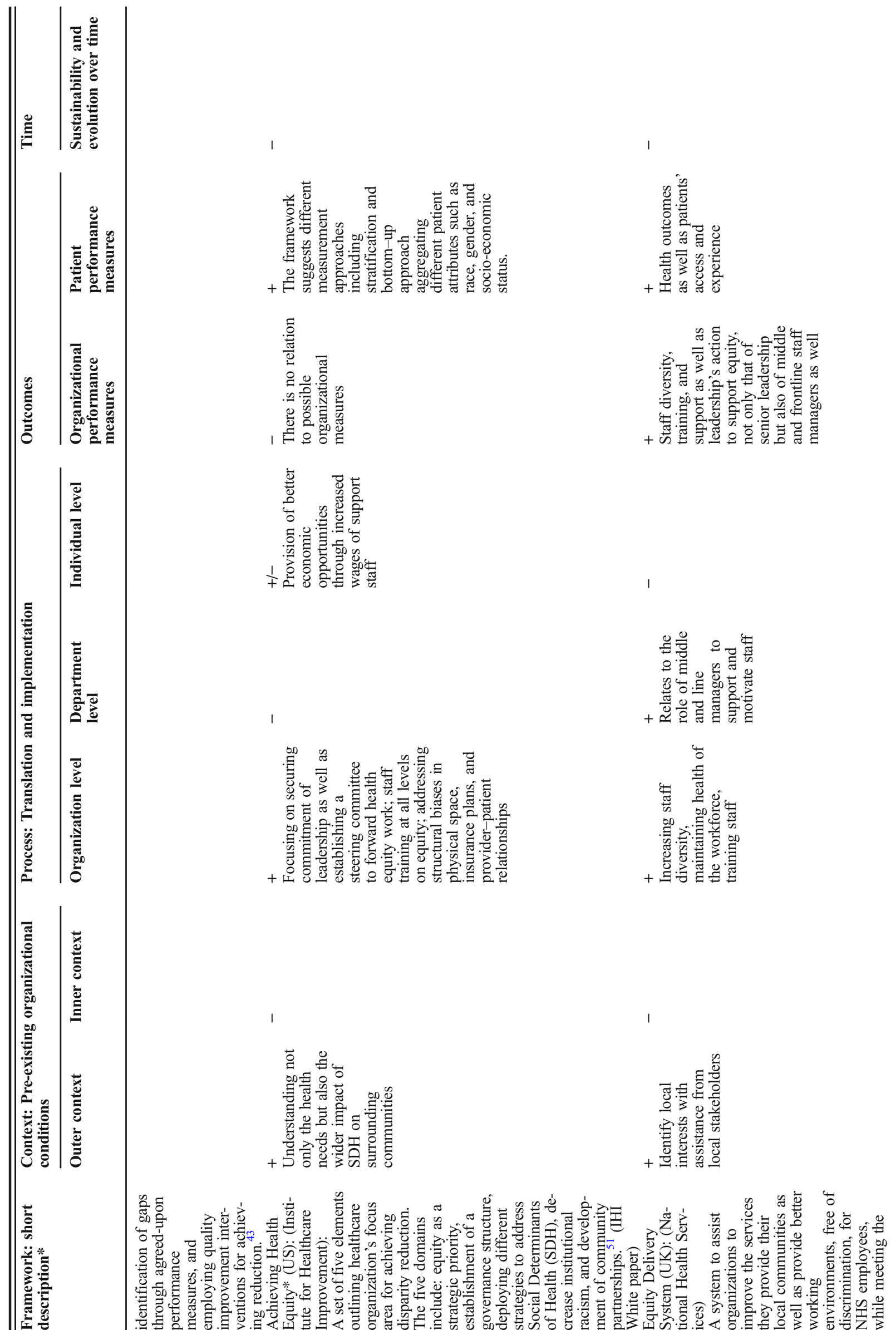


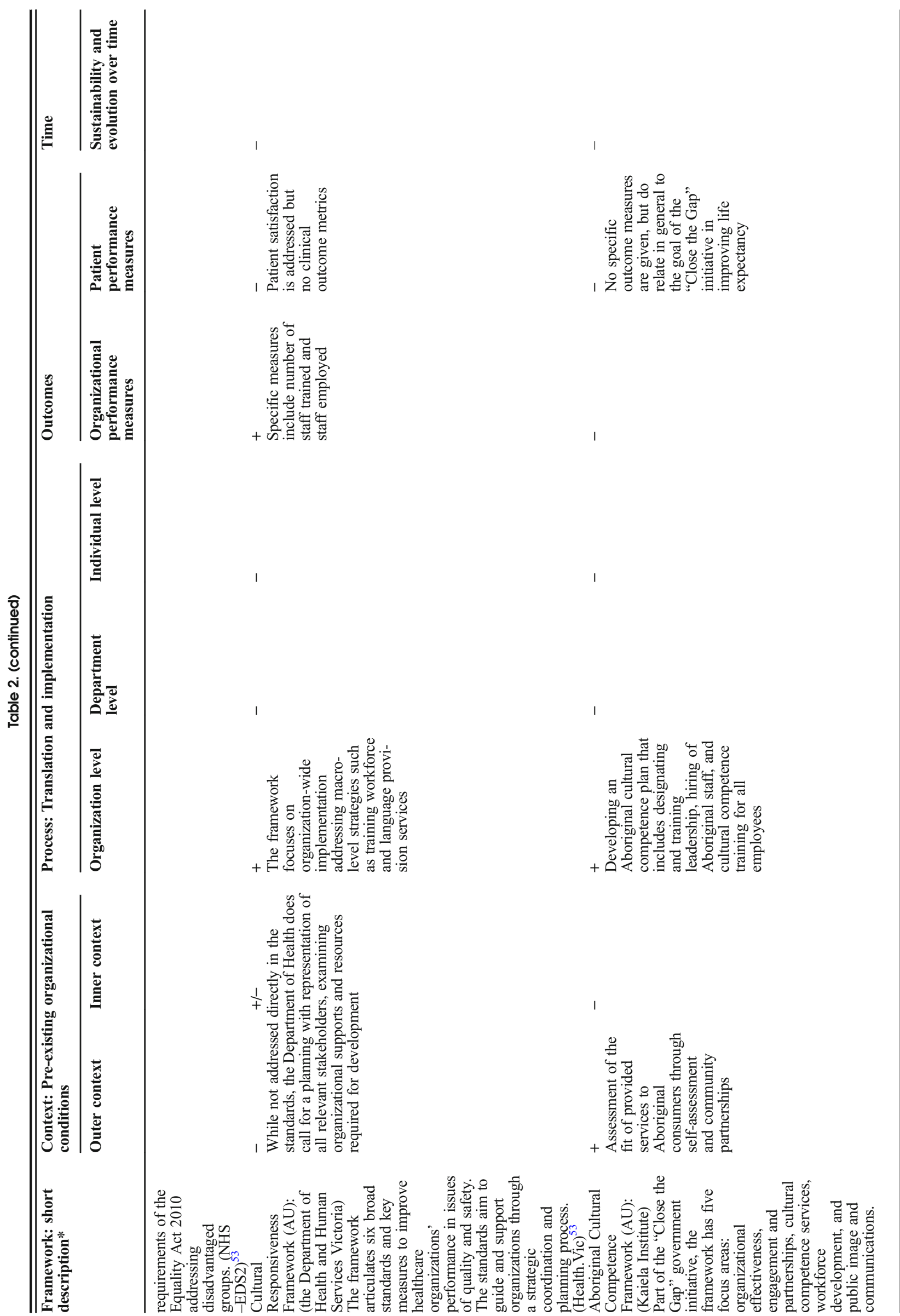



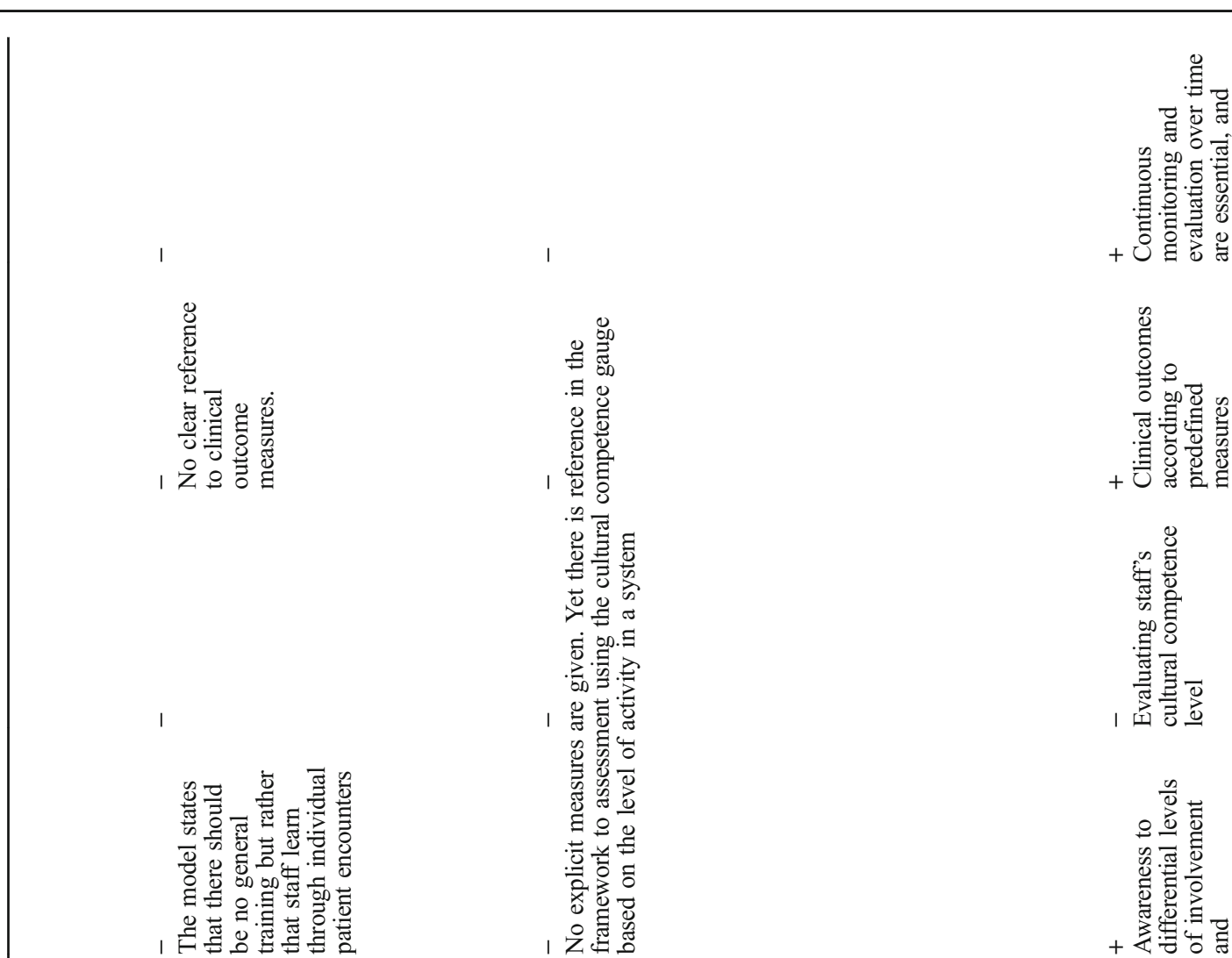

$+\circlearrowright \Xi \circlearrowright$

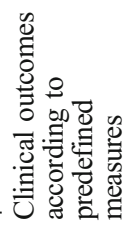

I
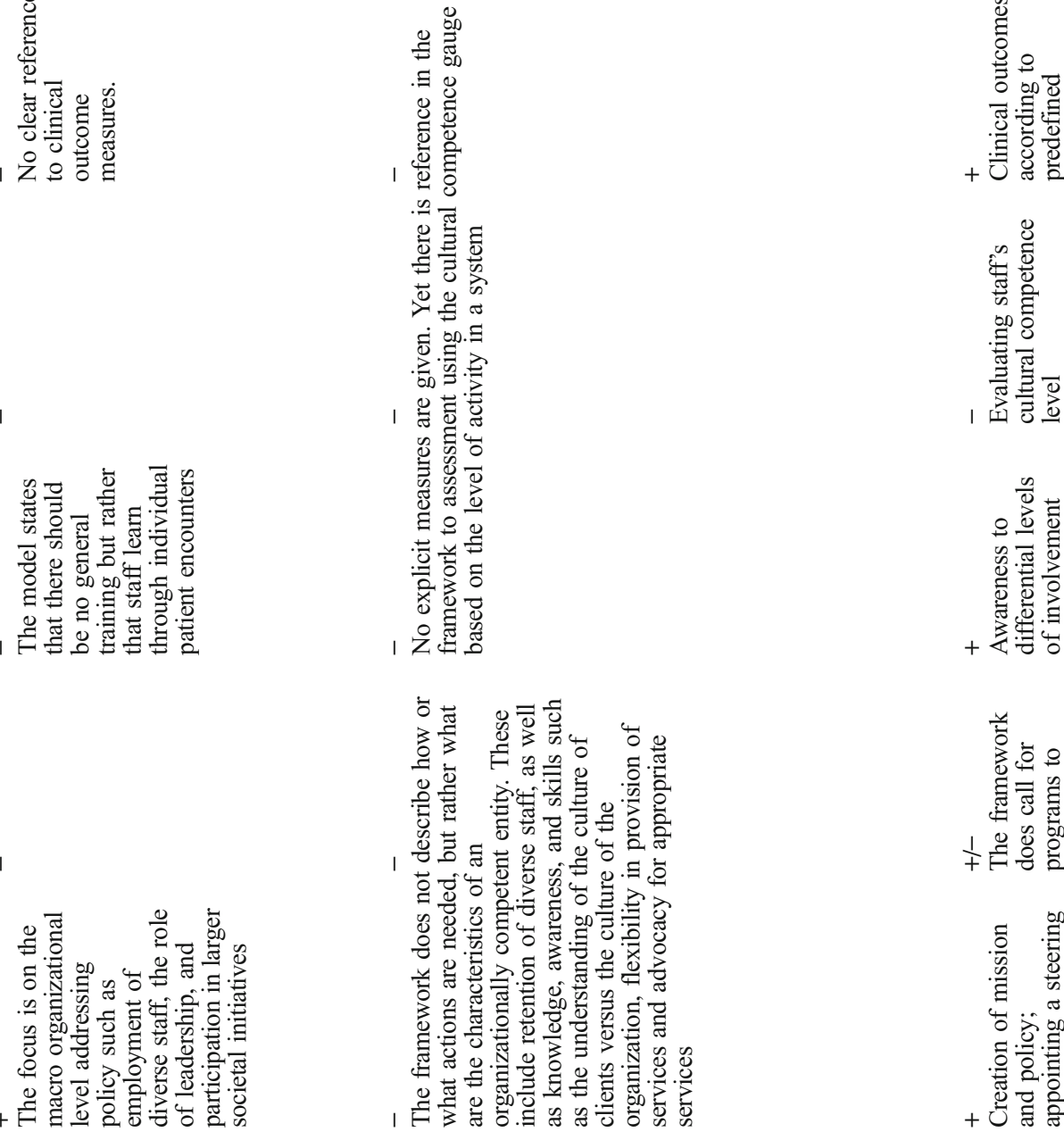

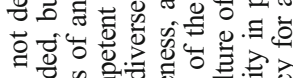

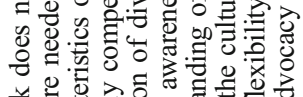

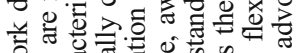

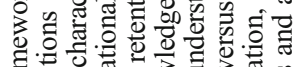

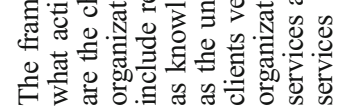
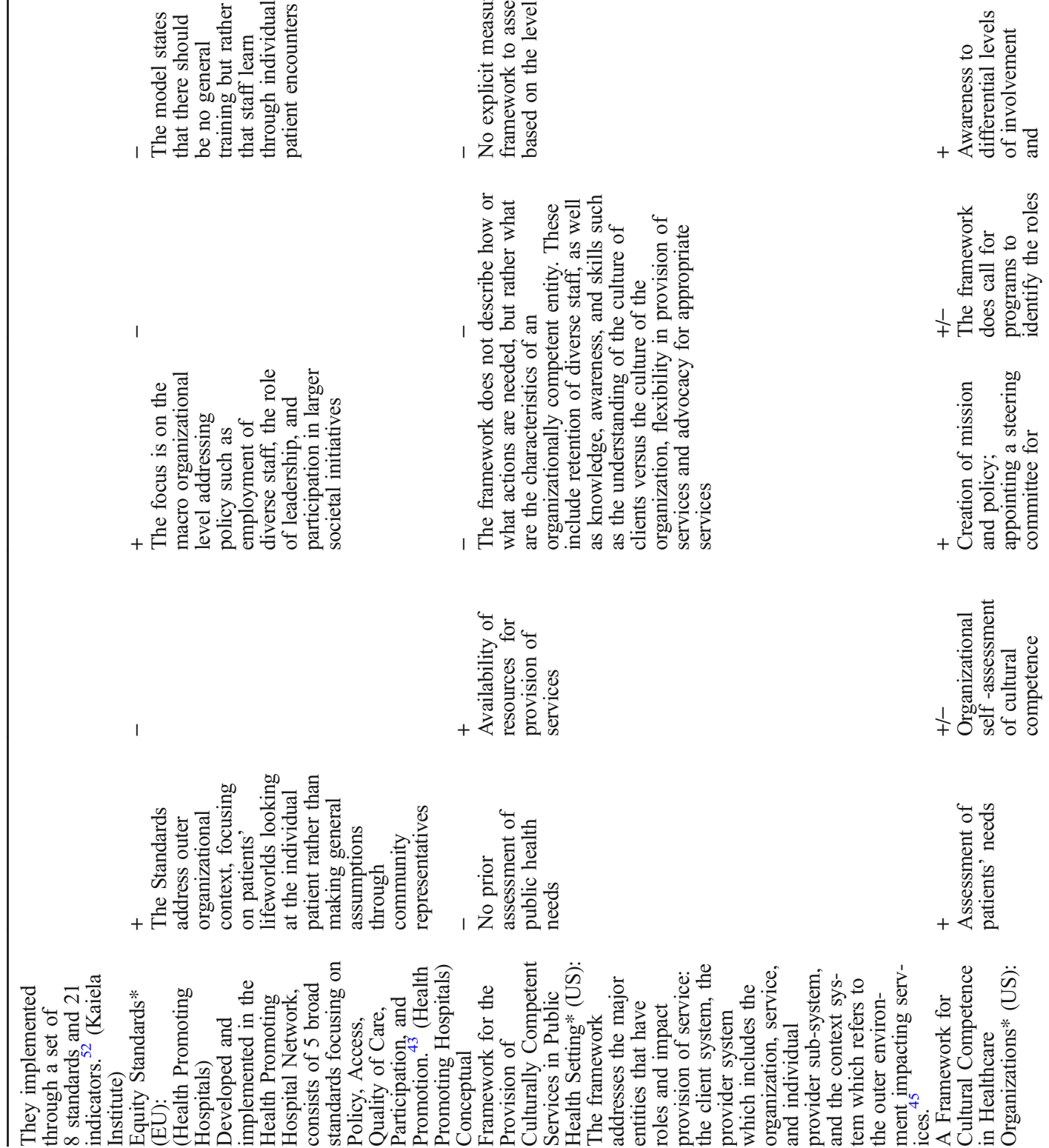


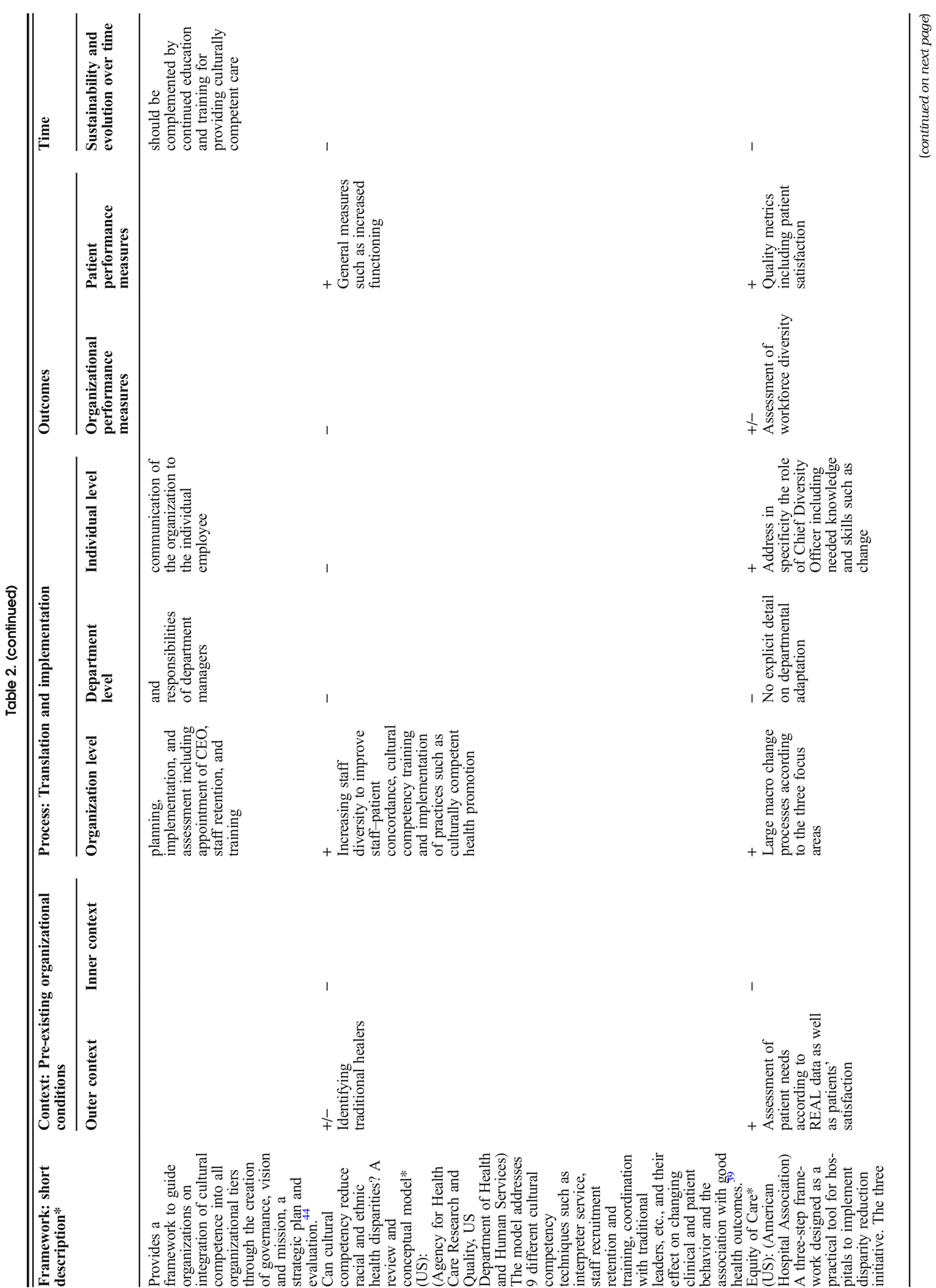


ncluded organization-wide training on cultural competence, leadership buy-in, and increasing workforce diversity. However, these guidelines do not specifically guide the tasks and skills required for successfully implementing the initiative throughout the differential intra-organizational departments. For example, the CLAS standard 9 states that an organization needs to: "Establish culturally and linguistically appropriate goals, policies and management accountability, and infuse them throughout the organization's planning and operations." However, it is not clear what are the specific processes and skills required for "infusing" equity, leaving the healthcare organization to interpret and devise the strategy on its own.

The Roadmap to Reduce Disparities model differs in its approach by creating a menu of intervention options based on analysis of six levels of influence, including provider, microsystem, and organization. This model offers change directives, not only on the macro but also on the meso and micro levels through initiatives such as team restructuring or tailored individual training. The menu of possibilities, rather than necessarily a whole-system approach, allows organizations to choose the extent of change they would like to implement, i.e., whether it be in a specific area of care or the organization at large. However, the translation process for implementing equity throughout the organization both across and within different departments is not addressed in detail.

Some reviewed models address the implementation process within specific micro-level employee groups. For example, the CLAS Standards refer specifically to the training of translators, the Equity Standards call for tailored clinical staff training around patient encounters, and the Achieving Health Equity framework calls for increased wages of support staff residing in surrounding disadvantaged communities. None of the frameworks or models address implementation at the meso department level. However, the Equality Delivery System model of the UK National Health Service, as well as the Disparities Leadership Program in the US, address the role of middle managers in supporting and motivating staff who are implementing equity-focused efforts.

\section{Outcomes}

Frameworks addressed clinical and patient experience performance measures but not measures of organizational culture or structure. Most models reference quality improvement equityfocused score cards, letting the organization decide which quality measures to evaluate. The Achieving Health Equity framework suggests measuring performance for individual sociodemographic attributes (e.g., black, female, low-income), combining the individual measurements into a summary index, and then comparing the index to the best health level among all groups as the reference. ${ }^{46}$

\section{Time}

Only three of the models address sustainability. The CLAS standards iterate the importance of continuously communicating achievements to relevant stakeholders as a broad and general 
guideline on sustainability. The Roadmap to Reduce Disparities calls for a proactive plan to ensure sustainability. The Framework for Cultural Competence in Health Care Organizations states the importance of continued monitoring, evaluation, and training of staff.

\section{DISCUSSION}

Overall, we found that current equity intervention frameworks do not fully address key issues relevant to successful implementation of organizational change. Existing models focus on assessing the outer organizational context mainly through analyzing racial and ethnic data and interacting with community representatives, implementing macro-level change processes such as cultural competence training of staff and increasing workforce diversity, and assessing patient outcomes.

Organizational theories stress the need for elasticity in the shape and pace of change. ${ }^{54}$ Assessment of the inner organizational context is needed to recognize possible barriers for implementation. Evaluation needs to include readiness for change of the organization, work group, and individuals, ${ }^{12,35}$ as resistance of employees can be a critical driver of failure. ${ }^{55}$ In their systematic review of cultural competence and improved patient outcomes, Truong and colleagues (2014) noted not only the sparse success of isolated cultural competence-based initiatives in impacting patient outcomes, but also the effect of organizational inner context on hindering implementation of these programs. ${ }^{56}$ Cultural competency training generally needs to be integrated with structural organizational change interventions to improve clinical performance measures. Additionally, to be most effective, organizations should engage in difficult discussions about how structural and institutional racism impacts their daily processes. ${ }^{57}$ Structural racism, such as residential segregation or care systems designed to preferentially attract affluent patients, systematically shapes healthcare access, utilization, and quality for racial and ethnic minority patients. ${ }^{58}$

The focus of existing frameworks on macro-level organizational implementation processes poses another challenge and area for improvement. Current guidelines are general and overarching, leaving the process of intra-organizational translation, adaptation, and implementation across different department and staff levels a black box for organizations to decipher. Middle managers entrusted with driving change and implementation often lack the knowledge and skills to effectively translate change processes. ${ }^{36,59}$ Parand and colleagues (2010) compared the perceptions of frontline and managerial staff on the factors important for successful implementation of a quality improvement initiative focused on quality and safety. They found that managers viewed learning sessions and training events to be the most important factors of the program, while frontline staff considered clinical and administrative systems and management support to be central to success. ${ }^{60}$ These findings highlight the complementary needs of managers and frontline staff. Managers seek training that would expand their knowledge and equip them with tangible skills for translating equity-focused organizational change to everyday work processes. Staff require a clear understanding of tasks required and organizational support systems to implement the initiative.

Studies detailing the implementation and evaluation of the CLAS framework, for example, have noted that most organizations implement the Standards partially. ${ }^{8,} 61,62$ WeechMaldonado and colleagues (2012) found that hospitals that adopted the CLAS Standards focused mainly on retaining translators and adapting culturally and language-appropriate materials. ${ }^{63}$ The standards pertaining to communication and language assistance are clear with specific change tasks, while other CLAS Standards are ambiguous. Ogbolu and Fitzpatrick (2015) argue that the sluggish adoption of the Standards stems from the difficulties of translating the Standards into policies and clinical practice across different healthcare settings. ${ }^{7}$

Finally, most existing equity frameworks do not adequately address sustainability and institutionalization of equityfocused change efforts. Sustainability will not occur without a conscious effort, addressing factors such as what values the organization truly prioritizes and how to create the business case for equity. ${ }^{30,64}$ Intervention practices and procedures are frequently abandoned due to "initiative decay" as organizational resources are diverted to other areas. ${ }^{37}$ Guidelines on institutionalization of equity-focused change initiatives need to include specific strategies for rollout, diffusion, and sustainability. ${ }^{65}$

\section{LIMITATIONS}

Our review did not include every patient term for social risk (e.g., migrant, refugee, and underserved) to the search filter for systematic reviews, and thus some equity frameworks may have been excluded. However, it is unlikely that the overall findings would be significantly different as this paper reviews models from multiple healthcare systems, countries, and contexts. Additionally, we may have misinterpreted or misclassified the frameworks and models. However, we were able to confirm the interpretations with authors of most of the models.

\section{CONCLUSIONS}

Existing inequity reduction frameworks and models lack important guidance to organizations for the practical implementation of change efforts, tending to focus on broad 30,000-foot statements. Several clear recommendations for improving future frameworks can be posited. First, frameworks should include guidelines on assessment of inner organizational context parameters, such as readiness for change and institutional racism, prior to implementation of change initiatives. Second, organizations require specific guidance on how to implement equity within and across all organizational levels. To effectively crack the black box of implementation, the trickle-down and translation of macro-level policies into the day-to-day 
action of frontline staff must be clear. Management personnel should receive training in "Translational Management," where they learn how to contextualize and implement equity in their specific departments. Finally, guidelines and strategies focusing on institutionalization and sustainability are crucial, considering competing organizational interests and changing environments. Providing organizations clear, effective, and concrete guidance on how to implement equity interventions has great potential for improving health equity.

Corresponding Author: Sivan Spitzer-Shohat, PhD; Department of Population Health, Azrieli Faculty of Medicine, Bar-llan University, Safed, Israel (e-mail: sivan.spitzer-shohat@biu.ac.il).

Funding Information Dr. Spitzer-Shohat was supported by a RivoEssrig Fellowship from the Department of Population Health, Azrieli Faculty of Medicine, Bar-Ilan University. Dr. Chin was partially supported by the Chicago Center for Diabetes Translation Research (grant number NIDDK P30 DK092949), the Robert Wood Johnson Foundation Finding Answers: Solving Disparities Through Payment and Delivery System Reform Program Office, and the Merck Foundation Bridging the Gap: Reducing Disparities in Diabetes Care National Program Office.

\section{Compliance with Ethical Standards:}

Conflict of Interest: The authors declare that they have no conflict of interest.

Publisher's Note: Springer Nature remains neutral with regard to jurisdictional claims in published maps and institutional affiliations.

\section{REFERENCES}

1. Clarke AR, Goddu AP, Nocon RS, et al. Thirty years of disparities intervention research: what are we doing to close racial and ethnic gaps in health care? Med Care. 2013;51(11):1020-1026. https://doi.org/10. 1097/MLR.0b013e3182a97ba3 [doi]

2. Chin MH. Quality improvement implementation and disparities: the case of the health disparities collaboratives. Med Care. 2010;48(8):668-675. https://doi.org/10.1097/MLR.0b013e3181e3585c [doi]

3. Balicer RD, Hoshen M, Cohen-Stavi C, et al. Sustained Reduction in Health Disparities Achieved through Targeted Quality Improvement: OneYear Follow-up on a Three-Year Intervention. Health Serv Res. 2015. https://doi.org/10.1111/1475-6773.12300 [doi]

4. Jha AK, Zaslavsky AM. Quality reporting that addresses disparities in health care. JAMA. 2014;312(3):225-226. https://doi.org/10.1001/ jama.2014.7204

5. Hunt B, Whitman S. Black: White Health Disparities in the United States and Chicago: 1990-2010. J Racial Ethn Heal Disparities 2015;2(1):93100. https://doi.org/10.1007/s40615-014-0052-0

6. McDonald KM, Schultz EM, Chang C. Evaluating the state of qualityimprovement science through evidence synthesis: insights from the closing the quality gap series. Perm J. 2013;17(4):52-61. https://doi. org/10.7812/TPP/13-010

7. Ogbolu Y, Fitzpatrick GA. Advancing Organizational Cultural Competency With Dissemination and Implementation Frameworks: Towards Translating Standards into Clinical Practice. ANS Adv Nurs Sci. 2015;38(3):203-214. https://doi.org/10.1097/ANS.0000000000000078

8. Barksdale CL, Rodick lii WH, Hopson R, Kenyon J, Green K, Jacobs CG. Literature Review of the National CLAS Standards: Policy and Practical Implications in Reducing Health Disparities. J Racial Ethn Heal Disparities. 2016. https://doi.org/10.1007/s40615-016-0267-3

9. Chin MH, Goldmann D. Meaningful Disparities Reduction Through Research and Translation Programs. JAMA. 2011;305(4):404. https:// doi.org/10.1001/jama.2011.26

10. Scott RW. Institutions and Organizations: Ideas, Interests, and Identities. 4th edn. SAGE Publications; 2013.
11. McNulty T, Ferlie E. Reengineering Health Care : The Complexities of Organizational Transformation. Oxford University Press; 2004.

12. Rafferty AE, Jimmieson NL, Armenakis AA. Change Readiness: A Multilevel Review. J Manage. 2013;39(1):110-135.

13. Jacobs G, Van Witteloostuijn A, Christe-Zeyse J. A theoretical framework of organizational change. J Organ Chang Manag. 2013;26(5):772-792. https://doi.org/10.1108/JOCM-09-2012-0137

14. Burnes B. Introduction: Why Does Change Fail, and What Can We Do About It? J Chang Manag. 2011;11(4):445-450. https://doi.org/10. 1080/14697017.2011.630507

15. Burnes B, Jackson P. Success and Failure In Organizational Change: An Exploration of the Role of Values. J Chang Manag. 2011;11(2):133-162. https://doi.org/10.1080/14697017.2010.524655

16. Warner Burke w. Organization change: theory and practice, 5th edn. Sage Publications; 2017.

17. Battilana J, Leca B, Boxenbaum E. How Actors Change Institutions: Towards a Theory of Institutional Entrepreneurship. Acad Manag Ann. 2009;3(1).

18. Ferlie E. Analysing health care organizations: a personal anthology. Routledge; 2016.

19. Benzer JK, Charns MP, Hamdan S, Afable M. The role of organizational structure in readiness for change: A conceptual integration. Heal Serv Manag Res. 2017;30(1):34-46. https://doi.org/10.1177/0951484816682396

20. Chin MH, Clarke AR, Nocon RS, et al. A roadmap and best practices for organizations to reduce racial and ethnic disparities in health care. J Gen Intern Med. 2012;27(8):992-1000. https://doi.org/10.1007/s11606012-2082-9 [doi]

21. Pettigrew AM, Woodman RW, Cameron KIMS. Studying Organizational Change and Development: for Future Research. 2001;44(4):697-713.

22. Poole MS, Van de Ven AH. Handbook of Organizational Change and Innovation. Oxford University Press; 2004.

23. Arksey H, O'Malley L. Scoping studies: towards a methodological framework. Int J Soc Res Methodol. 2005;8(1):19-32. https://doi.org/ 10.1080/1364557032000119616

24. Heather LC, Levac D, O'Brien KK, Straus S, Andrea CT, Perrier L, Kastner M, Moher D. Scoping reviews: time for clarity in definition, methods, and reporting. J Clin Epidemiol. 2014;67(12):1291-1294. https://doi.org/10.1016/J.JCLINEPI.2014.03.013

25. Anderson S, Allen P, Peckham S, Goodwin N. Asking the right questions: Scoping studies in the commissioning of research on the organisation and delivery of health services. Heal Res Policy Syst. 2008;6(1):7. https://doi.org/10.1186/1478-4505-6-7

26. Levac D, Colquhoun H, O'Brien KK. Scoping studies: advancing the methodology. Implement Sci. 2010;5:69. https://doi.org/10.1186/17485908-5-69

27. Brien SE, Lorenzetti DL, Lewis S, Kennedy J, Ghali WA. Overview of a formal scoping review on health system report cards. Implement Sci. 2010;5(1):2. https://doi.org/10.1186/1748-5908-5-2

28. Armenakis AA, Bedeian AG. Organizational Change: A Review of Theory and Research in the 1990s. J Manage. 1999;25(3):293-315. https://doi. org/10.1177/014920639902500303

29. Pettigrew AM. What is a processual analysis? Scand J Manag. 1997;13(4):337-348. https://doi.org/10.1016/S0956-5221(97)00020-1

30. DeMeester RH, Xu LJ, Nocon RS, Cook SC, Ducas AM, Chin MH. Solving Disparities Through Payment And Delivery System Reform: A Program To Achieve Health Equity. Health Aff. 2017;36(6):1133-1139. https://doi.org/10.1377/hlthaff.2016.0979

31. Albers Mohrman S, Tenkasi R V, Mohrman AM. The Role of Networks in Fundamental Organizational Change A Grounded Analysis. Role Netw J Appl Behav Sci. 2003;39(3):301-323. https://doi.org/10.1177/ 0021886303258072

32. Czarniwska B, Joerges B. Travels of Ideas. In: Czarniawska B, Sevon G, eds. Translating Organizational Change. New York: Walter de Gruyter; 1996: 13-48.

33. Maitlis S. The Social Processes of Organizational Sensemaking. Acad Manag J. 2005;48(1):21-49. http://www.jstor.org/stable/20159639

34. Weick KE. Sensemaking in Organizations. Thousand Oaks: Sage Publications; 1995.

35. Weiner BJ. A theory of organizational readiness for change. Implement Sci. 2009;4(1):67. https://doi.org/10.1186/1748-5908-4-67

36. Maitlis S, Christianson M. Sensemaking in Organizations: Taking Stock and Moving Forward. Acad Manag Ann. 2014;8(1).

37. Buchanan D, Fitzgerald L, Ketley D, et al. No going back: A review of the literature on sustaining organizational change. Int J Manag Rev. 2005;7(3): 189-205. https://doi.org/10.1111/j.1468-2370.2005. $00111 . \mathrm{x}$ 
38. Andrew B, Sutton Anthea PD. Systematic Approaches to a Successful Literature Review. SAGE Publications; 2016.

39. Brach C, Fraser I. Can cultural competency reduce racial and ethnic health disparities? A review and conceptual model. Med Care Res Rev. 2000;57 Suppl 1:181-217. https://doi.org/10.1177/1077558700057001S09

40. Betancourt JR, Green AR, Carrillo JE, et al. Defining Cultural Competence: A Practical Framework for Addressing Racial/Ethnic Disparities in Health and Health Care. Public Health Rep. 2003;118(4):293. https://doi.org/10.1093/phr/118.4.293

41. Betancourt JR, Tan-McGrory A, Kenst KS, Phan TH, Lopez L. Organizational Change Management For Health Equity: Perspectives From The Disparities Leadership Program. Health Aff (Millwood). 2017;36(6):1095-1101. https://doi.org/10.1377/hlthaff.2017.0022

42. National Quality Forum. A roadmap for promoting health equity and elimiting disparities. The Four I's for Health Equity; 2017. http://www. qualityforum.org/Home.aspx. Accessed September 2017

43. Cattacin S, Chiarenza A, Domenig D. Equity standards for healthcare organisations: a theoretical framework. Divers Equal Heal Care. 2013;10(4):249-258.

44. Castillo RJ, Guo KL. A framework for cultural competence in health care organizations. Health Care Manag (Frederick). 2011;30(3):205-214. doi:https://doi.org/10.1097/HCM.0b013e318225dfe6

45. Thompson-Robinson M, Reininger B, Sellers B, Saunders R, Davis $\mathbf{K}$, Ureda J. Conceptual framework for the provision of culturally competent services in public health settings. J Cult Divers. 2006;13(2):97-104.

46. Wyatt R, Laderman M, Botwinick L, Mate $\mathbf{K}$, Whittington J. Acheiving Health Equity: A Guide for Healthcare Organizations. IHI White Paper. Cambridge; 2016. ihi.org. Accessed September 2017

47. Health Research Educational Trust. Equity of care: a toolkit for eliminating health care disparities ${ }^{\circledR}$. 2015.

48. Office of Minority Health. National standards for CLAS in health and health care: a blueprint for advancing and sustaining CLAS policy and practice. 2013. https://www.thinkculturalhealth.hhs.gov/pdfs/EnhancedCLASStandardsBlueprint.pdf\#page82. Accessed September 2017

49. National Health Service. A refreshed equality delivery system for the NHS: EDS2 making sure that everyone counts. 2013. https://www.england. nhs.uk/wp-content/uploads/2013/11/eds-nov131.pdf. Accessed September 2017

50. Rural and Regional Health and Aged Care Services. Cultural responsiveness framework. 2009. www.health.vic.gov.au/cald. Accessed September 2017

51. Laderman M, Whittington J. A Framework for improving Health Equity. Healthc Exec. 2016;31(3):82,84-85

52. Tynan M, Smullen F, Atkinson P, Stephens K. Aboriginal cultural competence for health services in regional Victoria: lessons for implementation. Aust New Zeal J Public Heal. 2013;37(4):392-393.
53. Seeleman C, Essink-Bot M-L, Stronks K, Ingleby D. How should health service organizations respond to diversity? A content analysis of six approaches. BMC Health Serv Res. 2015;15:510. https://doi.org/10. 1186/s12913-015-1159-7

54. Batras D, Duff C, Smith BJ. Organizational change theory: implications for health promotion practice. Health Promot Int. 2014;129(5 Pt 1):dau098. https://doi.org/10.1093/heapro/dau098

55. Weiner BJ, Amick H, Lee S-YD. Review: Conceptualization and Measurement of Organizational Readiness for Change. Med Care Res Rev. 2008;65(4):379-436. https://doi.org/10.1177/1077558708317802

56. Truong $\mathbf{M}$, Paradies $\mathbf{Y}$, Priest N. Interventions to improve cultural competency in healthcare: a systematic review of reviews. BMC Health Serv Res. 2014;14(1):99. https://doi.org/10.1186/1472-6963-14-99

57. Chin MH, King PT, Jones RG, et al. Lessons for achieving health equity comparing Aotearoa/New Zealand and the United States. Health Policy. 2018;0(0). https://doi.org/10.1016/j.healthpol.2018.05.001

58. Bailey ZD, Krieger N, Agénor M, Graves J, Linos N, Bassett MT Structural racism and health inequities in the USA: evidence and interventions. Lancet. 2017;389(10077):1453-1463. https://doi.org/10. 1016/S0140-6736(17)30569-X

59. LUScher LS, Lewis MW. Organizational Change and Managerial Sensemaking: Working Through Paradox. Acad Manag J. 2008;51(2):221-240. https://doi.org/10.5465/AMJ.2008.31767217

60. Parand A, Burnett S, Benn J, Pinto A, Iskander S, Vincent C. The disparity of frontline clinical staff and managers' perceptions of a quality and patient safety initiative. J Eval Clin Pract. 2011;17(6):1184-1190. https://doi.org/10.1111/j.1365-2753.2010.01506.x

61. Diamond LC, Wilson-Stronks A, Jacobs EA. Do hospitals measure up to the national culturally and linguistically appropriate services standards? Med Care. 2010;48(12):1080-1087. https://doi.org/10.1097/MLR. Ob013e3181f380bc

62. Gómez ML, Charnigo R, Harris TT, Williams JC, Pfeifle W. Assessment of National CLAS Standards in Rural and Urban Local Health Departments in Kentucky. J Public Health Manag Pract. 2016;22(6):576-585. https://doi.org/10.1097/PHH.0000000000000410

63. Weech-Maldonado R, Dreachslin JL, Brown J, et al. Cultural competency assessment tool for hospitals: evaluating hospitals' adherence to the culturally and linguistically appropriate services standards. Health Care Manage Rev. 2012;37(1):54-66. https://doi.org/10.1097/HMR. Ob013e31822e2a4f

64. Chin MH. Creating the Business Case for Achieving Health Equity. J Gen Intern Med. 2016;31(7):792-796. https://doi.org/10.1007/s11606-0163604-7

65. Moore JE, Mascarenhas A, Bain J, Straus SE. Developing a comprehensive definition of sustainability. Implement Sci. 2017;12(1):110. https://doi.org/10.1186/s13012-017-0637-1 